\title{
Generalization of Transitive Cayley Digraphs
}

\author{
Anil Kumar V. ${ }^{1} \&$ Mohanan T. ${ }^{2}$ \\ ${ }^{1}$ Department of Mathematics, University of Calicut, Malappuram, Kerala, India \\ ${ }^{2}$ Department of Mathematics, Sree Narayana Guru College, Kozhikode, Kerala, India \\ Correspondence: Anil Kumar V., Department of Mathematics, University of Calicut, Malappuram, Kerala 673 \\ 635, India. E-mail: anilashwin2003@yahoo.com
}

Received: September 25, 2012 Accepted: October 15, 2012 Online Published: November 21, 2012

doi:10.5539/jmr.v4n6p43 URL: http://dx.doi.org/10.5539/jmr.v4n6p43

\begin{abstract}
This paper proves several extended theoretical results of transitive Cayley digraphs. Several generalization of transitive Cayley digraphs also have been provided. Moreover, various graph properties have been expressed in terms of algebraic properties. This did not attract much attention in the literature.
\end{abstract}

Keywords: Cayley digraphs, transitive digraphs, quasi-transitive digraphs, $k$-transitive digraphs, $k$-quasi-transitive digraphs

\section{AMS Subject Classification number (2000): 05C25}

\section{Introduction}

A binary relation on a set $V$ is a subset $E$ of $V \times V$. A digraph is a pair $(V, E)$ where $V$ is a nonempty set (called vertex set) and $E$ is a binary relation on $V$. The elements of $E$ are the edges of the digraph. An edge of the form $(x, x)$ is called a loop. Through out this paper we assume that $G$ is a digraph without loops. A $k$ - chain or a path of length $k$, is a sequence $\left(x_{0}, x_{1}, \ldots, x_{k}\right)$ of vertices, each adjacent to its successor, and all the internal vertices $x_{1}, x_{2}, \ldots, x_{k-1}$ are distinct. A digraph $G$ is said to be transitive if $E^{2} \subseteq E$, that is, $(x, y),(y, z) \in E$ implies $(x, z) \in E$. A digraph is quasi-transitive if $(x, y),(y, z) \in E$ implies $(x, z) \in E$ or $(z, x) \in E$ (Galeana-Sanchez \& Cesar Hernandez-Cruz, 2011). Observe that every transitive digraph is quasi-transitive.

Let $G$ be a group and let $S$ be a subset of G. The Cayley digraph of $G$ with respect to $S$ is defined as the digraph $X=(G, E)$, where $E$ is a subset of $G \times G$, such that $(x, y) \in E$ if and only if $x^{-1} y \in S$ (Dobson, 2006). The Cayley digraph of $G$ with respect to $S$ is denoted by $\operatorname{Cay}(G, S)$. The subset $S$ is called the connection set of $X$. That is, Cayley digraph $\operatorname{Cay}(G, S)$ has as its vertex-set and edge-set, respectively, $V=G$ and $E=\{(x, y)$ : $y=x z$ for some $z \in S\}$. Observe that transitive/quasi-transitive Cayley digraphs are special classes of Cayley digraphs and these Cayley digraphs play an import role in algebraic graph theory. In this paper, we introduce some generalization of transitive/quasi-transitive Cayley digraphs. Moreover, we express various graph properties in terms of algebraic properties.

\section{2. k-(quasi-) Transitive Cayley Digraphs}

The concepts of $k$-transitive and $k$-quasi transitive digraphs were first introduced by Galeana-Sanchez and Cesar Hernandez-Cruz (2011). Observe that the above concepts are generalizations of transitive and quasi-transitive digraphs respectively. The aim of this section is to characterize $k$-transitive/ $k$-quasi-transitive Cayley digraphs. We start with the following definitions due to Galeana-Sanchez and Cesar Hernandez-Cruz (2011).

Definition 2.1 A digraph $G$ is $k$ - transitive if the existence of a directed path $\left(x_{0}, x_{1}, \ldots, x_{k}\right)$ of length $k$ in $G$ implies that $\left(x_{0}, x_{k}\right) \in E$.

Definition 2.2 A digraph $G$ is $k$-quasi-transitive if, whenever $\left(x_{0}, x_{1}, \ldots, x_{k}\right)$ is a directed path of length $k$, then $\left(x_{0}, x_{k}\right) \in E$ or $\left(x_{k}, x_{0}\right) \in E$.

Here we prove that $\operatorname{Cay}(G, S)$ is $k$-transitive if and only if $S^{k} \subseteq S$ and $k$-quasi-transitive if and only if $S^{k} \subseteq S \cup S^{-1}$. We also provide examples of $k$-transitive/ $k$-quasi-transitive Cayley digraphs.

Theorem 2.3 Cay $(G, S)$ is $k$-transitive if and only if $S^{k} \subseteq S$. 
Proof. Assume that $\operatorname{Cay}(G, S)$ is $k$-transitive. Let $x \in S^{k}$. Then there exists $x_{1}, x_{2}, \ldots, x_{k} \in S$ such that

$$
x=x_{1} x_{2} \ldots x_{k}
$$

Let $z_{0}=1, z_{1}=x_{1}, z_{2}=x_{1} x_{2}, \ldots, z_{k-1}=x_{1} x_{2} \ldots x_{k-1}$. Consider the following ordered pairs of elements in $G$ :

$$
\left(z_{0}, z_{1}\right),\left(z_{1}, z_{2}\right),\left(z_{2}, z_{3}\right), \ldots,\left(z_{k-1}, x\right)
$$

We see that

$$
\begin{aligned}
z_{0}^{-1} z_{1}= & 1^{-1} x_{1} \in S \Rightarrow\left(z_{0}, z_{1}\right) \in E, \\
z_{1}^{-1} z_{2}= & x_{1}^{-1} x_{1} x_{2}=x_{2} \in S \Rightarrow\left(z_{1}, z_{2}\right) \in E, \\
& \vdots \\
z_{k-1}^{-1} x= & \left(x_{1} x_{2} \cdots x_{k-1}\right)^{-1}\left(x_{1} x_{2} \cdots x_{k}\right)=x_{k} \in S \Rightarrow\left(z_{k-1}, x\right) \in E .
\end{aligned}
$$

Hence $\left(z_{0}, z_{1}, z_{2}, \ldots, z_{k-1}, x\right)$ is a directed path of length $k$ from 1 to $x$. Since $\operatorname{Cay}(G, S)$ is $k$ - transitive, $(1, x)$ is an edge in Cay $(G, S)$. In other words $x \in S$. Hence $S^{k} \subseteq S$.

Conversely, assume that $S^{k} \subseteq S$. Let $\left(x_{0}, x_{1}, \ldots, x_{k}\right)$ be a directed path of length $k$ in $\operatorname{Cay}(G, S)$. This implies that $x_{0}^{-1} x_{1}, x_{1}^{-1} x_{2}, x_{2}^{-1} x_{3}, \ldots, x_{k-1}^{-1} x_{k} \in S$. Equivalently,

$$
\left(x_{0}^{-1} x_{1}\right)\left(x_{1}^{-1} x_{2}\right)\left(x_{2}^{-1} x_{3}\right) \ldots\left(x_{k-1}^{-1} x_{k}\right) \in S^{k}
$$

In other words $x_{0}^{-1} x_{k} \in S$. This implies that $\left(x_{0}, x_{k}\right)$ is an edge in $\operatorname{Cay}(G, S)$. Hence $\operatorname{Cay}(G, S)$ is $k$-transitive.

Corollary 2.4 Cay $(G, S)$ is 2-transitive(that is, transitive) if and only if $S^{2} \subseteq S$.

Theorem 2.5 $\operatorname{Cay}(G, S)$ is $k$-quasi-transitive if and only if $S^{k} \subseteq S \cup S^{-1}$.

Proof. Assume that $\operatorname{Cay}(G, S)$ is $k$-quasi-transitive. Let $x \in S^{k}$. Then there exists $x_{1}, x_{2}, \ldots, x_{k}$ such that $x=$ $x_{1} x_{2} \ldots x_{k}$. Note that

$$
\left(1, x_{1}, x_{1} x_{2}, \ldots, x_{1} x_{2} \ldots x_{k-1}, x\right)
$$

is a directed path of length $k$ from 1 to $x$. Since $\operatorname{Cay}(G, S)$ is $k$ - transitive, either $(1, x)$ or $(x, 1)$ is an edge in $\operatorname{Cay}(G, S)$. In other words $x \in S \cup S^{-1}$. Hence $S^{k} \subseteq S \cup S^{-1}$.

Conversely, assume that $S^{k} \subseteq S \cup S^{-1}$. Let $\left(x_{0}, x_{1}, \ldots, x_{k}\right)$ be a directed path of length $k$ in $\operatorname{Cay}(G, S)$. Then, $x_{0}^{-1} x_{1}, x_{1}^{-1} x_{2}, x_{2}^{-1} x_{3}, \ldots, x_{k-1}^{-1} x_{k} \in S$. Equivalently,

$$
\left(x_{0}^{-1} x_{1}\right)\left(x_{1}^{-1} x_{2}\right)\left(x_{2}^{-1} x_{3}\right) \ldots\left(x_{k-1}^{-1} x_{k}\right) \in S^{k}
$$

In other words $x_{0}^{-1} x_{k} \in S \cup S^{-1}$. This implies that either $\left(x_{0}, x_{k}\right)$ or $\left(x_{k}, x_{0}\right)$ is an edge in $\operatorname{Cay}(G, S)$. Hence $\operatorname{Cay}(G, S)$ is $k$-quasi-transitive.

Corollary 2.6 Cay $(G, S)$ is 2-quasi transitive(that is, quasi-transitive) if and only if $S^{2} \subseteq S \cup S^{-1}$.

The following are some examples of $k$ - transitive/ $k$-quasi transitive Cayley digraphs.

Example 2.7 Let $k$ be any positive integer greater than or equal to 2. Let $S=\left\{k, k^{2}, 2 k^{2}-k, 3 k^{2}-2 k, \ldots\right\}$. Then the Cayley digraph, $\operatorname{Cay}(\mathbb{Z}, S)$ is an infinite $k$-transitive Cayley digraph.

Example 2.8 Let $\mathbb{Z}_{6}$ denotes the cyclic group of order 6 . Then $\operatorname{Cay}\left(\mathbb{Z}_{6},\{2,5\}\right)$ and $\operatorname{Cay}\left(\mathbb{Z}_{6},\{4,1\}\right)$ are 4-transitive Cayley digraphs (see Figure 1).

Example 2.9 Consider the permutation group $S_{3}=\{(1),(123),(132),(23),(12),(13)\}$. Let $S=\{(23),(12),(13)\}$. Then Cay $\left(S_{3}, S\right)$ is a 3-transitive Cayley digraph.

Example 2.10 Let $\mathbb{Z}_{8}$ denotes the cyclic group of order 8. Then the Cayley digraphs, Cay $\left(\mathbb{Z}_{8},\{1,3\}\right)$ and Cay $\left(\mathbb{Z}_{8},\{1,5\}\right)$ are 3-quasi-transitive Cayley digraphs (see Figure 2). 

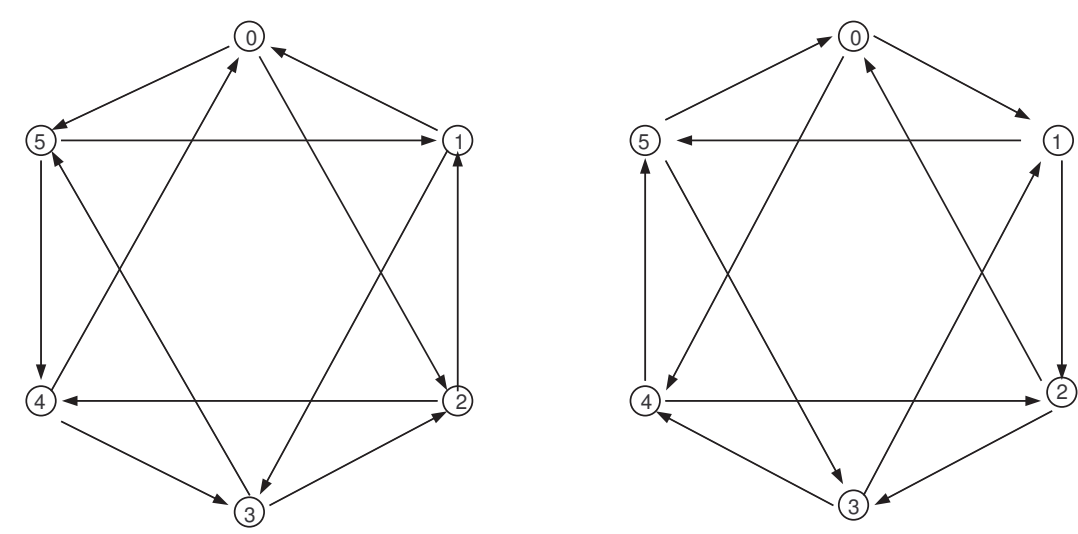

Figure 1. Cay $\left(\mathbb{Z}_{6},\{2,5\}\right)$ and $\operatorname{Cay}\left(\mathbb{Z}_{6},\{4,1\}\right)$
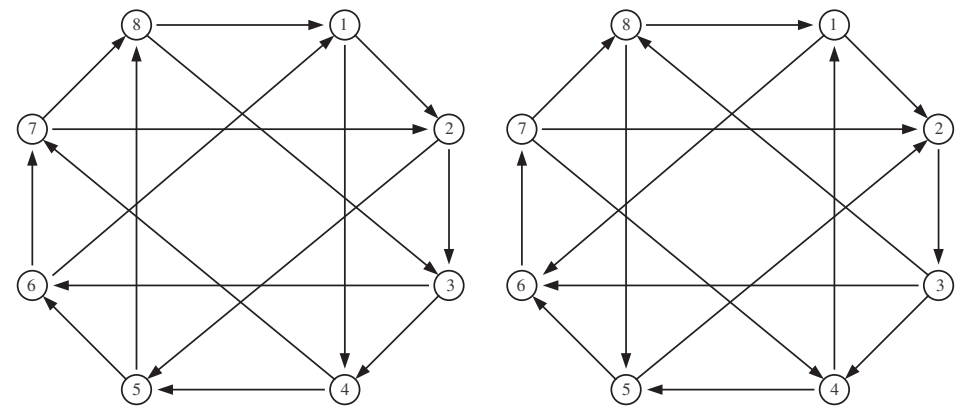

Figure 2. $\operatorname{Cay}\left(\mathbb{Z}_{8},\{1,3\}\right)$ and $\operatorname{Cay}\left(\mathbb{Z}_{8},\{1,5\}\right)$
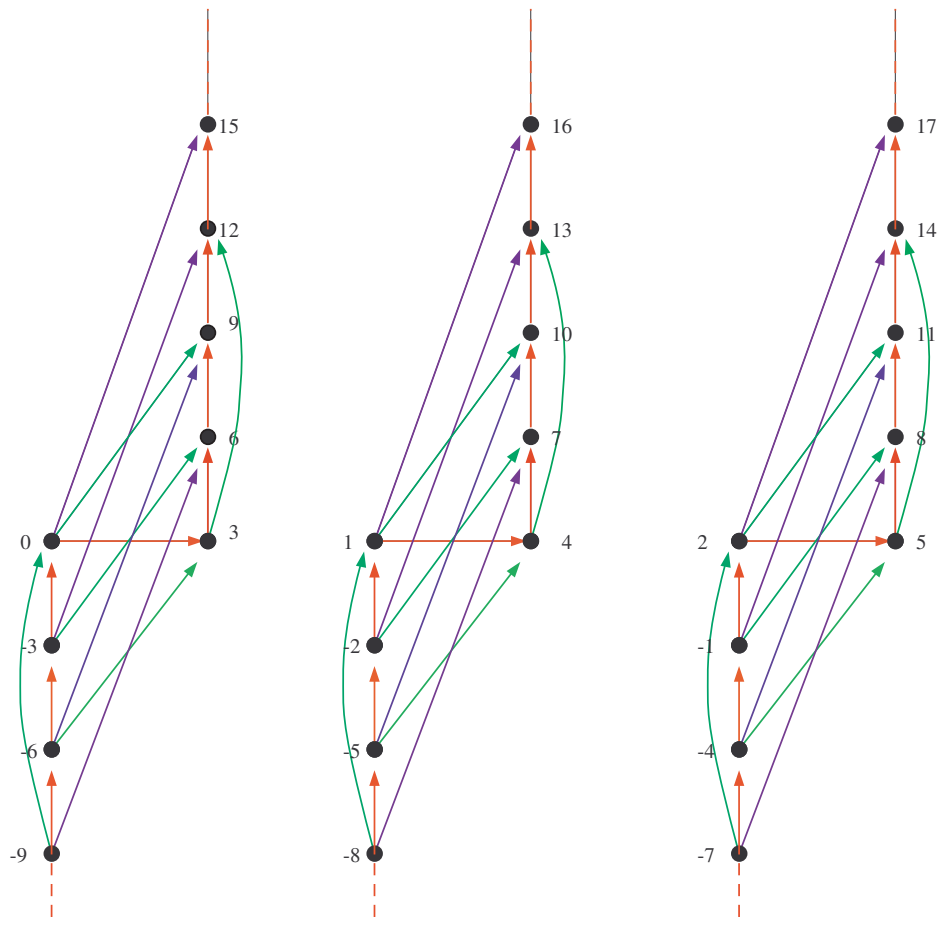

Figure 3. A portion of $\operatorname{Cay}(\mathbb{Z},\{3,9,15,21, \ldots\})$ 


\section{Remark 2.11}

1) Any 2- transitive(that is, transitive) Cayley digraph is $k$-transitive for any positive integer $k$ greater than equal to 3. But the converse need not be true. For example, $\operatorname{Cay}\left(\mathbb{Z}_{6},\{2,5\}\right)$ is 4-transitive but not 2-transitive.

2) $\operatorname{Cay}(\mathbb{Z},\{3,9,15,21, \ldots\})$ is $2 n+1$-transitive for each $n \in \mathbb{N}$.

3) $\operatorname{Cay}(\mathbb{Z},\{3,9,15,21, \ldots\})$ is the disjoint union of three copies of the Cayley digraph: $\operatorname{Cay}(3 \mathbb{Z},\{3,9,15,21, \ldots\})$ (see Figure 3).

4) Let $k$ be any positive integer greater than or equal to 2 . Let $S=\left\{k, k^{2}, 2 k^{2}-k, 3 k^{2}-2 k, \ldots\right\}$. Then the Cayley digraph, $\operatorname{Cay}(\mathbb{Z}, S)$ is a disjoint union of $k, k$-transitive digraphs.

Theorem 2.12 Any infinite $k$-transitive Cayley digraph $\operatorname{Cay}(G, S)$ is $n(k-1)+k$-transitive for every $n \in \mathbb{N}$.

Proof. Assume that $\operatorname{Cay}(G, S)$ be $k$-transitive. We will prove the result by induction on $n$. For $n=1$, consider $S^{k+k-1}$. Observe that $S^{k+k-1}$ can be written as:

$$
S^{k+k-1}=S^{k} S^{k-1} \subseteq S S^{k-1}=S^{k} \subseteq S .
$$

Next, assume that the theorem is true for $n=r$. Hence $S^{r(k-1)+k} \subseteq S$. We will show that the result is true for $n=r+1$. For, it suffices to show that $S^{(r+1)(k-1)+k} \subseteq S$. Note that $S^{(r+1)(k-1)+k}$ can be written as:

$$
\begin{aligned}
S^{(r+1)(k-1)+k} & =S^{[r(k-1)+k]+(k-1)} \\
& =S^{[r(k-1)+k]} S^{k-1} \subseteq S S^{k-1}=S^{k} \subseteq S .
\end{aligned}
$$

Hence the result follows by mathematical induction.

Theorem 2.13 Cay $(G, S)$ is $k$-(quasi-) transitive if and only if $C a y\left(G, S^{-1}\right)$ is $k$-(quasi-) transitive.

Proof. First, assume that $\operatorname{Cay}(G, S)$ is $k$-transitive. Let $\left(x_{0}, x_{1}, \ldots, x_{k}\right)$ be a directed path of length $k$ in $\operatorname{Cay}\left(G, S^{-1}\right)$. This implies that

$$
x_{0}^{-1} x_{1}, x_{1}^{-1} x_{2}, x_{2}^{-1} x_{3}, \ldots, x_{k-1}^{-1} x_{k} \in S^{-1} .
$$

Equivalently, $x_{1}^{-1} x_{0}, x_{2}^{-1} x_{1}, x_{3}^{-1} x_{2}, \ldots, x_{k}^{-1} x_{k-1} \in S$. This tells us that

$$
\left(x_{k}, x_{k-1}, \ldots, x_{1}, x_{0}\right)
$$

is a path of length $k$ in $\operatorname{Cay}(G, S)$. Since $\operatorname{Cay}(G, S)$ is $k$-transitive, $x_{k}^{-1} x_{0} \in S$. That is $x_{0}^{-1} x_{k} \in S^{-1}$. Hence $\left(x_{0}, x_{k}\right)$ is an edge in $\operatorname{Cay}\left(G, S^{-1}\right)$. The converse is straightforward.

Definition 2.14 Let $G$ be a digraph. The least positive integer $k$ such that $G$ is $k$-transitive is called the $k$-transitivity number of $G$, denoted by $\mathscr{A}(G)$. That is,

$$
\mathscr{A}(G):=\min \{k \in \mathbb{N}: G \text { is } k \text { - transitive }\} .
$$

If no such $k$ exists, we define $\mathscr{A}(G)=0$. In a similar manner we can define the $k$-quasi- transitivity number of $G$ as follows:

$$
\mathscr{B}(G)=\min \{k \in \mathbb{N}: G \text { is } k \text { - quasi- transitive }\} .
$$

We define $\mathscr{B}(G)=0$, if no such $k$ exists.

Theorem 2.15 The k-transitivity number of $\operatorname{Cay}(G, S)$ is the least positive integer $k$ such that $S^{k} \subseteq S$. That is,

$$
\mathscr{A}(\operatorname{Cay}(G, S))=\min \left\{k \in \mathbb{N}: S^{k} \subseteq S\right\} .
$$

Theorem 2.16 The k-quasi-transitivity number of $\operatorname{Cay}(G, S)$ is the least positive integer $k$ such that $S^{k} \subseteq S \cup S^{-1}$. That is,

$$
\mathscr{B}(\operatorname{Cay}(G, S))=\min \left\{k \in \mathbb{N}: S^{k} \subseteq S \cup S^{-1}\right\} .
$$

The transitivity/quasi-transitivity numbers of some Cayley digraphs are given below:

$$
\begin{aligned}
& \text { (i) } \mathscr{A}\left(\operatorname{Cay}\left(\mathbb{Z}_{6},\{2,5\}\right)\right)=4, \mathscr{A}((\operatorname{Cay}(\mathbb{Z},\{3,9,15,21, \ldots\}))=3, \\
& \text { (ii) } \mathscr{B}\left(\operatorname{Cay}\left(\mathbb{Z}_{8},\{1,3\}\right)\right)=3, \mathscr{B}\left(\operatorname{Cay}\left(\mathbb{Z}_{8},\{1,5\}\right)\right)=3 .
\end{aligned}
$$


Theorem 2.17 Let $C a y(G, S)$ be a cayley digraph with connection set $S$. Then we have the following:

$$
\begin{aligned}
\text { (i) } \mathscr{A}(\operatorname{Cay}(G, S)) & =\mathscr{A}\left(\operatorname{Cay}\left(G, S^{-1}\right)\right) \\
\text { (ii) } \mathscr{B}(\operatorname{Cay}(G, S)) & =\mathscr{B}\left(\operatorname{Cay}\left(G, S^{-1}\right)\right) .
\end{aligned}
$$

\section{3. k-path-(quasi-) Transitive Cayley Digraphs}

Galeana-Sanchez and Cesar Hernandez-Cruz (2011) generalized the definition of transitive digraphs as follows:

Definition 3.18 A digraph $G$ is called $k$-path transitive if whenever there is a $x y$ directed path of length less than or equal to $k$ and a $y z$ directed path of length less than or equal to $k$, then there exists a $x z$-directed path of length less than or equal to $k$.

We define the $k$-path transitivity number of a digraph $G$ as follows:

$$
\mathscr{C}(G):=\min \{k \in \mathbb{N}: G \text { is } k \text { path transitive }\} .
$$

We define $\mathscr{C}(G)=0$, if no such $k$ exists.

Theorem 3.19 $\operatorname{Cay}(G, S)$ is k-path-transitive if and only if $\left(S \cup S^{2} \cup \cdots S^{k}\right)^{2} \subseteq S \cup S^{2} \cdots \cup S^{k}$.

Proof. First assume that $\operatorname{Cay}(G, S)$ is $k$-path-transitive. Let $x \in\left(S \cup S^{2} \cup \cdots S^{k}\right)^{2}$. Then $x=z_{1} z_{2}$ for some $z_{1}, z_{2} \in\left(S \cup S^{2} \cup \cdots S^{k}\right)$. This implies that there exists a path of length less than or equal to $k$ from 1 to $z_{1}$ and a path of length less than or equal to $k$ from $z_{1}$ to $x$. Since $\operatorname{Cay}(G, S)$ is $k$-path-transitive there exists a path of length less than or equal to $k$ from 1 to $x$. This implies that $x \in\left(S \cup S^{2} \cup \cdots S^{k}\right)$. Hence $\left(S \cup S^{2} \cup \cdots S^{k}\right)^{2} \subseteq S \cup S^{2} \cdots \cup S^{k}$.

Converse is straight forward.

Corollary 3.20 Cay $(G, S)$ is 1-path-transitive (that is, transitive) if and only if $S^{2} \subseteq S$.

Theorem 3.21 For any Cayley digraph $\operatorname{Cay}(G, S)$, we have $\mathscr{C}(\operatorname{Cay}(G, S))=\min \left\{k \in \mathbb{N}:\left(S \cup S^{2} \cup \cdots S^{k}\right)^{2} \subseteq\right.$ $\left.S \cup S^{2} \cdots \cup S^{k}\right\}$.

We define the following:

Definition 3.22 A digraph $G$ is called $k$-path-quasi-transitive if whenever there is a $x y$ directed path of length less than or equal to $k$ and a $y z$ directed path of length less than or equal to $k$, then there exists a $x z$-directed path of length less than or equal to $k$ or there exists a $z x$-directed path of length less than or equal to $k$. We define $k$-path-quasi-transitivity number of $G$ as:

$$
\mathscr{D}(G)=\min \{k \in \mathbb{N}: G \text { is } k \text { - quasi-path- transitive }\} .
$$

Theorem 3.23 Cay $(G, S)$ is k-path-quasi-transitive if and only if $\left(S \cup S^{2} \cup \cdots S^{k}\right)^{2} \subseteq\left(S \cup S^{-1}\right) \cup\left(S^{2} \cup\left(S^{-1}\right)^{2}\right) \cdots \cup$ $\left(S^{k} \cup\left(S^{-1}\right)^{k}\right)$.

Corollary 3.24 Cay $(G, S)$ is 1-path-quasi-transitive(that is, quasi-transitive) if and only if $S^{2} \subseteq S \cup S^{-1}$.

Theorem 3.25 Cay $(G, S)$ is k-path-quasi-transitive if and only if $C a y\left(G, S^{-1}\right)$ is k-path-quasi-transitive.

Theorem 3.26 For any Cayley digraph $\operatorname{Cay}(G, S), \mathscr{D}(\operatorname{Cay}(G, S))$ is given by

$$
\mathscr{D}(\operatorname{Cay}(G, S))=\min \left\{k \in \mathbb{N}:\left(S \cup S^{2} \cup \cdots S^{k}\right)^{2} \subseteq\left(S \cup S^{-1}\right) \cup\left(S^{2} \cup\left(S^{-1}\right)^{2}\right) \cdots \cup\left(S^{k} \cup\left(S^{-1}\right)^{k}\right)\right\} .
$$

Theorem 3.27 Let Cay $(G, S)$ be a Cayley digraph with connection set $S$. Then we have the following:

$$
\begin{aligned}
\text { (i) } \mathscr{C}(\operatorname{Cay}(G, S)) & =\mathscr{C}\left(\operatorname{Cay}\left(G, S^{-1}\right)\right) \\
\text { (ii) } \mathscr{D}(\operatorname{Cay}(G, S)) & =\mathscr{D}\left(\operatorname{Cay}\left(G, S^{-1}\right)\right) .
\end{aligned}
$$

\section{4. (m, n)-(quasi-) Transitive Cayley Digraphs}

In this section we generalize the definition of $k$-transitive and $k$-quasi-transitive digraphs as follows:

Definition 4.28 Let $m$ and $n$ be two positive integers such that $m>n$. A digraph $G$ is ( $m, n)$-transitive whenever there is a directed path of length $m$ from $x$ to $y$ there is a directed path of length $n$ from $x$ to $y$. We define the 
$(m, n)$-transitivity number of a digraph as the least positive integers $m$ and $n$ such that $G$ is $(m, n)$-transitive. That is,

$$
\mathscr{E}(G)=\min _{m, n}\{(m, n) \in \mathbb{N} \times \mathbb{N}, m>n: G \text { is }(m, n)-\text { transitive }\} .
$$

We define, $\mathscr{E}(G)=(0,0)$, if no such $m$ and $n$ exist.

Definition 4.29 Let $m$ and $n$ be two positive integers such that $m>n$. A digraph $G$ is $(m, n)$-quasi-transitive whenever there is a directed path of length $m$ from $x$ to $y$ there is a directed path of length $n$ from $x$ to $y$ or a directed path of length $n$ from $y$ to $x$. We define the $(m, n)$-quasi-transitivity number of a digraph as the least positive integers $m$ and $n$ such that $G$ is $(m, n)$-quasi-transitive. That is,

$$
\mathscr{F}(G)=\min _{m, n}\{(m, n) \in \mathbb{N} \times \mathbb{N}, m>n: G \text { is }(m, n)-\text { quasi transitive }\} .
$$

If no such $m$ and $n$ exist, we define $\mathscr{F}(G)=(0,0)$.

Theorem 4.30 $\operatorname{Cay}(G, S)$ is $(m, n)$-transitive if and only if $S^{m} \subseteq S^{n}$.

Proof. First assume that $\operatorname{Cay}(G, S)$ is $(m, n)$-transitive. Let $x \in S^{m}$. Then

$$
x=z_{1} z_{2} \cdots z_{m} \text { for some } z_{1}, z_{2}, \ldots, z_{m} \in S .
$$

Let $x_{0}=1, x_{1}=z_{1}, x_{2}=z_{1} z_{2}, \ldots, x_{m}=x$. This implies that the sequence of vertices: $\left(x_{0}, x_{1}, x_{2}, \ldots, x_{m}\right)$ is a path of length $m$ from $x_{0}$ to $x$. Since $\operatorname{Cay}(G, S)$ is $(m, n)$ - transitive, there is a path of length $n$, say, $\left(1, y_{1}, y_{2}, \ldots, y_{n-1}, x\right)$ from 1 to $x$. This implies that $x \in S^{n}$. Hence $S^{m} \subseteq S^{n}$.

Conversely, assume that $S^{m} \subset S^{n}$. We will show that $\operatorname{Cay}(G, S)$ is $(m, n)$-transitive. Let $\left(x_{0}, x_{1}, x_{2}, \ldots, x_{m}\right)$ be a path of length $m$ from $x_{0}$ to $x_{m}$. This implies that $x_{0}^{-1} x_{1}, x_{1}^{-1} x_{2}, \ldots, x_{m-1}^{-1} x_{m} \in S$. That is

$$
\left(x_{0}^{-1} x_{1}\right)\left(x_{1}^{-1} x_{2}\right) \ldots\left(x_{m-1}^{-1} x_{m}\right)=x_{0}^{-1} x_{m} \in S^{m} \subseteq S^{n}
$$

This implies that

$$
x_{0}^{-1} x_{m}=t_{1} t_{2} \ldots t_{n} \text { for some } t_{i} \in S
$$

This implies that $\left(1, t_{1}, t_{1} t_{2}, \ldots, t_{1} t_{2} \ldots t_{n}\right)$ is a path of length $n$ form 1 to $t_{1} t_{2} \ldots t_{n}$. That is,

$$
\left(x_{0}, x_{0} t_{1}, x_{0} t_{1} t_{2}, \ldots, x_{0} t_{1} t_{2} \ldots t_{n}\right)
$$

is a path of length of $n$ from $x_{0}$ to $x_{m}$. Hence $\operatorname{Cay}(G, S)$ is $(m, n)$-transitive.

Corollary 4.31 $\operatorname{Cay}(G, S)$ is $(k, 1)$-transitive (that is, $k$-transitive) if and only if $S^{k} \subseteq S$.

Corollary 4.32 Cay $(G, S)$ is $(2,1)$-transitive (that is, transitive) if and only if $S^{2} \subseteq S$.

Theorem 4.33 $\operatorname{Cay}(G, S)$ is $(m, n)$-quasi-transitive if and only if $S^{m} \subseteq S^{n} \cup\left(S^{-1}\right)^{n}$.

Corollary 4.34 Cay $(G, S)$ is $(k, 1)$-quasi-transitive (that is, $k$-quasi-transitive) if and only if $S^{k} \subseteq S \cup S^{-1}$.

Corollary 4.35 Cay $(G, S)$ is $(2,1)$-quasi-transitive (that is, quasi-transitive) if and only if $S^{2} \subseteq S \cup S^{-1}$.

Theorem 4.36 The $(m, n)$-transitivity number of $\operatorname{Cay}(G, S)$ is the least positive integers $m, n(m>n)$ such that $S^{m} \subseteq S^{n}$. That is,

$$
\mathscr{F}(\operatorname{Cay}(G, S))={ }_{m, n}^{\min }\left\{(m, n) \in \mathbb{N} \times \mathbb{N}, m>n: S^{m} \subseteq S^{n}\right\} .
$$

Theorem 4.37 The $(m, n)$-quasi-transitivity number of $\operatorname{Cay}(G, S)$ is the least positive integers $m, n(m>n)$ such that $S^{m} \subseteq S^{n} \cup\left(S^{-1}\right)^{n}$. That is,

$$
\mathscr{F}(\operatorname{Cay}(G, S))={ }_{m, n}^{\min }\left\{(m, n) \in \mathbb{N} \times \mathbb{N}, m>n: S^{m} \subseteq S^{n} \cup\left(S^{-1}\right)^{n}\right\} .
$$

\section{5. (m, n)-path-(quasi-) Transitive Cayley Digraphs}

Definition 5.38 Let $m$ and $n$ be two positive integers such that $m \geq n$. A digraph $G$ is called ( $m, n)$-path-transitive if whenever there is a $x y$ directed path of length less than or equal to $m$ and a $y z$ directed path of length less than or equal to $m$, then there exists a $x z$-directed path of length less than or equal to $n$. We define the $(m, n)$-pathtransitivity number of $G$ as:

$$
\mathscr{G}(G)=\min _{m, n}\{(m, n) \in \mathbb{N} \times \mathbb{N}, m \geq n: G \text { is }(m, n) \text { path transitive }\} .
$$


Also, define $\mathscr{G}(G)=(0,0)$, if no such numbers $m$ and $n$ exist.

Theorem 5.39 Cay $(G, S)$ is $(m, n)$-path-transitive if and only if $\left(S \cup S^{2} \cup \cdots S^{m}\right)^{2} \subseteq\left(S \cup S^{2} \cup \cdots S^{n}\right)$.

Corollary 5.40 Cay $(G, S)$ is $(k, k)$-path-transitive if and only if $\left(S \cup S^{2} \cup \cdots S^{k}\right)^{2} \subseteq\left(S \cup S^{2} \cup \cdots S^{k}\right)$.

Corollary $5.41 \operatorname{Cay}(G, S)$ is $(k, 1)$-path-transitive if and only if $\left(S \cup S^{2} \cup \cdots S^{k}\right)^{2} \subseteq S$.

Corollary $5.42 \operatorname{Cay}(G, S)$ is $(1,1)$-path-transitive if and only if $S^{2} \subseteq S$.

Theorem 5.43 The $(m, n)$-path-transitivity number of the $(m, n)$-path-transitive Cayley digraph Cay $(G, S)$ is the least positive integers $m, n(m \geq n)$ such that $\left(S \cup S^{2} \cup \cdots S^{m}\right)^{2} \subseteq\left(S \cup S^{2} \cup \cdots S^{n}\right)$. That is,

$$
\mathscr{G}(\operatorname{Cay}(G, S))=\min _{m, n}\left\{(m, n) \in \mathbb{N} \times \mathbb{N}, m \geq n:\left(S \cup S^{2} \cup \cdots S^{m}\right)^{2} \subseteq\left(S \cup S^{2} \cup \cdots S^{n}\right)\right\} .
$$

Definition 5.44 Let $m$ and $n$ be two positive integers such that $m \geq n$. A digraph $G$ is called $(m, n)$-path-quasitransitive if whenever there is a $x y$ directed path of length less than or equal to $m$ and a $y z$ directed path of length less than or equal to $m$, then there exists a $x z$-directed path of length less than or equal to $n$ or there exists a $z x$-directed path of length less than or equal to $n$. We define the $(m, n)$-quasi-transitive number of $G$ as:

$$
\mathscr{H}(G)=\min _{m, n}\{(m, n) \in \mathbb{N} \times \mathbb{N}, m \geq n: G \text { is }(m, n) \text {-quasi-path transitive }\} .
$$

Theorem $5.45 \operatorname{Cay}(G, S)$ is $(m, n)$-path-quasi-transitive if and only if

$$
\left(S \cup S^{2} \cup \cdots S^{m}\right)^{2} \subseteq\left(S \cup S^{-1}\right) \cup\left(S^{2} \cup\left(S^{-1}\right)^{2}\right) \cup \cdots\left(S^{n} \cup\left(S^{-1}\right)^{n}\right) .
$$

Corollary $5.46 \operatorname{Cay}(G, S)$ is $(k, k)$-path-quasi-transitive if and only if

$$
\left(S \cup S^{2} \cup \cdots S^{k}\right)^{2} \subseteq\left(S \cup S^{-1}\right) \cup\left(S^{2} \cup\left(S^{-1}\right)^{2}\right) \cup \cdots\left(S^{n} \cup\left(S^{-1}\right)^{k}\right) .
$$

Corollary 5.47 Cay $(G, S)$ is (k,1)-path-quasi-transitive if and only if $\left(S \cup S^{2} \cup \cdots S^{k}\right)^{2} \subseteq S \cup S^{-1}$.

Corollary $5.48 \operatorname{Cay}(G, S)$ is $(1,1)$-path-transitive if and only if $S^{2} \subseteq S \cup S^{-1}$.

Theorem 5.49 The $(m, n)$-path-quasi-transitivity number of the digraph $\operatorname{Cay}(G, S)$ is the least positive integers $m, n(m \geq n)$ such that $\left(S \cup S^{2} \cup \cdots S^{m}\right)^{2} \subseteq\left(S \cup S^{-1}\right) \cup\left(S^{2} \cup\left(S^{-1}\right)^{2}\right) \cup \cdots\left(S^{n} \cup\left(S^{-1}\right)^{n}\right)$. That is, $\mathscr{I}($ Cay $(G, S))$ is given by

$$
\mathscr{I}(\operatorname{Cay}(G, S))=\min _{m, n}\left\{(m, n) \in \mathbb{N} \times \mathbb{N}, m>n:\left(S \cup \cdots \cup S^{m}\right)^{2} \subseteq\left(S \cup S^{-1}\right) \cup \cdots \cup\left(S^{n} \cup\left(S^{-1}\right)^{n}\right)\right\} .
$$

\section{6. m-n-(quasi)-transitive Transitive Cayley Digraphs}

In this section we define the following:

Definition 6.50 Let $m$ and $n$ be positive integers such that $m>n$. A digraph $G$ is $m-n$ transitive whenever there is a directed path of length $m$ from $x$ to $y$ there is a directed path of length almost $n$ from $x$ to $y$. We define the $m$ - $n$ transitivity number of $G$ as:

$$
\mathscr{J}(G)=\min _{m, n}\{(m, n) \in \mathbb{N} \times \mathbb{N}, m>n: G \text { is } m-n \text { transitive }\} .
$$

Also, define $\mathscr{J}(G)=(0,0)$, if no such numbers $m$ and $n$ exist.

Theorem 6.51 $\operatorname{Cay}(G, S)$ is $m$-n transitive if and only if $S^{m} \subseteq S \cup S^{2} \cdots \cup S^{n}$.

Corollary $6.52 \operatorname{Cay}(G, S)$ is $k$-1 transitive(that is, $k$-transitive) if and only if $S^{k} \subseteq S$.

Corollary 6.53 Cay $(G, S)$ is 2-1 transitive (that is, transitive) if and only if $S^{2} \subseteq S$.

Definition 6.54 A digraph $G$ is $m$-n-quasi-transitive whenever there is a directed directed path of length $m$ from $x$ to $y$ there is a directed path of length almost $n$ from $x$ to $y$ or there is a directed path of length almost $n$ from $y$ to $x$. We define the $m$-n-quasi-transitivity number of $G$ as:

$$
\mathscr{K}(G)=\min _{m, n}\{(m, n) \in \mathbb{N} \times \mathbb{N}, m>n: G \text { is } m-n \text { quasi- transitive }\} .
$$


Also, define $\mathscr{K}(G)=(0,0)$, if no such numbers $m$ and $n$ exist.

Theorem 6.55 Cay $(G, S)$ is m-n transitive if and only if $S^{m} \subseteq\left(S \cup S^{-1}\right) \cup\left(S^{2} \cup\left(S^{-1}\right)^{2}\right) \cdots \cup S^{n} \cup\left(S^{-1}\right)^{n}$.

Corollary 6.56 $\operatorname{Cay}(G, S)$ is $k$-1 quasi-transitive(that is, $k$-quasi-transitive) if and only if $S^{k} \subseteq S \cup S^{-1}$.

Corollary 6.57 Cay $(G, S)$ is 2-1-transitive (that is, quasi-transitive) if and only if $S^{2} \subseteq S$.

\section{7. k-(quasi-)hasse Diagrams}

We define the following:

Definition 7.58 A digraph $G$ is a $k$-hasse diagram if the existence of a directed path $\left(x_{0}, x_{1}, \ldots, x_{k}\right)$ of length $k$ in $G$ implies that $\left(x_{0}, x_{k}\right) \notin E$.

We prove the following:

Theorem 7.59 Cay $(G, S)$ is a k-hasse diagram if and only if $S^{k} \cap S=\emptyset$.

Proof. First, assume that $S^{k} \cap S=\emptyset$. Let $\left(x_{0}, x_{1}, \ldots, x_{k}\right)$ be path of length $k$ in $G$. Then we have $x_{0}^{-1} x_{1}, x_{1}^{-1} x_{2}, \ldots, x_{k-1} x_{k} \in$ $S$. This implies that

$$
\left(x_{0}^{-1} x_{1}\right)\left(x_{1}^{-1} x_{2}\right) \ldots\left(x_{k-1} x_{k}\right)=x_{0}^{-1} x_{k} \in S^{k}
$$

This implies that $x_{0}^{-1} x_{k} \notin S$. Hence $\left(x_{0}, x_{k}\right)$ is not an edge in $\operatorname{Cay}(G, S)$.

Conversely, suppose that $\operatorname{Cay}(G, S)$ is a $k$-hasse diagram. Assume that $x \in S^{k}$. Then $x=z_{1} z_{2} \cdots z_{k}$ for some $z_{1}, z_{2}, \ldots, z_{k} \in S$. This implies that $\left(1, z_{1}, z_{1} z_{2}, \ldots, x\right)$ is a path of length $k$ in $\operatorname{Cay}(G, S)$. Since $\operatorname{Cay}(G, S)$ is a $k$-Hasse diagram, we have $(1, x) \notin S$. This implies that $x \notin S$. Hence $S^{k} \cap S=\emptyset$.

Definition 7.60 A digraph $G$ is called a hasse diagram if and only if $G$ is a $k$-hasse diagram for every $k \geq 2$.

Theorem 7.61 Cay $(G, S)$ is a hasse diagram if and only if $S^{k} \cap S=\emptyset$ for every $k \geq 2$.

Definition 7.62 A digraph $G$ is a $k$-strong-hasse diagram if the existence of a directed path $\left(x_{0}, x_{1}, \ldots, x_{k}\right)$ of length $k$ in $G$ implies that $\left(x_{0}, x_{k}\right) \notin E$ and $\left(x_{k}, x_{0}\right) \notin E$.

Theorem 7.63 $\operatorname{Cay}(G, S)$ is a $k$-strong-hasse diagram if and only if $S^{k} \cap S=\emptyset$ and $1 \notin S^{k}$.

Definition 7.64 A digraph $G$ is called a strong-hasse diagram if and only if it is $k$-strong-hasse diagram for every $k \geq 2$.

Theorem 7.65 $\operatorname{Cay}(G, S)$ is a strong-hasse diagram if and only if $S^{k} \cap S=\emptyset$ and $1 \notin S^{k}$ for every $k \geq 2$.

We define the following:

Definition 7.66 A digraph $G$ is called an anti- $k$-hasse diagram if the existence of a directed path $\left(x_{0}, x_{1}, \ldots, x_{k}\right)$ of length $k$ in $G$ implies that there exists some $i(2 \leq i \leq k)$ such that $\left(x_{0}, x_{i}\right) \in E$. We define the anti-k-hasse index of $G$ as:

$$
\mathscr{L}(G)=\min \{k \in \mathbb{N}: G \text { is an } k \text { anti-hasse diagram }\} .
$$

Sampathkumarachar et al. (2010) generalized the definition of transitive digraphs as follows:

Definition 7.67 A digraph $G$ is said to be $k$ - transitive if whenever $\left(x_{0}, x_{1}, \ldots, x_{k}\right)$ is a directed path of length $k$, then there exists an integer $i, 2 \leq i \leq k$ such that $\left(x_{0}, x_{i}\right) \in E$.

This definition is obviously, a generalization of transitive graphs. Observe that the definitions due to Sampathkumarachar et al. (2010) and Galeana-Sanchez and Cesar Hernandez-Cruz (2011), are entirely different even though they used the same term "k- transitive digraph". In this paper, we use the term "anti- $k$-hasse diagram" instead of $k$-transitive in the definition due to Sampathkumarachar et al. to avoid confusion.

We define the following:

Definition 7.68 A digraph $G$ is anti-k-quasi-hasse diagram if the existence of a directed path $\left(x_{0}, x_{1}, \ldots, x_{k}\right)$ of length $k$ in $G$ implies that there exists some $i(2 \leq i \leq k)$ such that or $\left(x_{0}, x_{i}\right) \in E$ or $\left(x_{i}, x_{0}\right) \in E$. We define the anti- $k$-quasi-hasse index of $G$ as:

$$
\mathscr{M}(G)=\min \{k \in \mathbb{N}: G \text { is an anti } k \text {-quasi-hasse diagram }\} .
$$

Theorem 7.69 Cay $(G, S)$ is an anti-k-hasse diagram if and only if there exists an integer $i(2 \leq i \leq k)$, such that $S^{i} \subseteq S$. 
Proof. Assume that $S^{i} \subseteq S$ for some $i$. Let $\left(x, x_{1}, x_{2}, \ldots, y\right)$ be a directed path of length $k$ from $x$ to $y$. Then we have the following sets of equations:

$$
\begin{gathered}
x_{1}=x t_{1}, \\
x_{2}=x_{1} t_{2}, \\
x_{3}=x_{2} t_{3}, \\
\vdots \\
y=x_{k-1} t_{k} .
\end{gathered}
$$

for some $t_{1}, t_{2}, \ldots, t_{k} \in S$. From Equation (2), $x_{i}$ can be written as

$$
x_{i}=x t_{1} t_{2} \ldots t_{i}
$$

Let $t=t_{1} t_{2} \ldots t_{i}$. Then we have $x_{i}=x t$ where $t \in S^{i} \subseteq S$. This implies that $\left(x, x_{i}\right)$ is an edge in $\operatorname{Cay}(G, S)$. Hence $\operatorname{Cay}(G, S)$ is an anti $k$-hasse diagram.

Conversely, assume that $\operatorname{Cay}(G, S)$ is an anti $k$-hasse diagram. For $i=1,2, \ldots k$, let $x_{i}$ be an arbitrary element in $S^{i}$. Then $x_{i}=t_{1} t_{2} \ldots t_{i}$ for some $t_{i} \in S$. Let $x=t_{k} t_{k-1} \ldots t_{2} t_{1}$. Then $x$ is an arbitrary element in $S^{k}$. Note that $\left(1, x_{1}, x_{2}, \ldots, x\right)$ is a directed path leading from 1 to $x$. Then there exists an integer $i$ such that $\left(1, x_{i}\right)$ is an edge in Cay $(G, S)$. This implies that $x_{i} \in S$. Since $x_{i}$ is an arbitrary element in $S^{i}$, we have $S^{i} \subseteq S$.

Corollary 7.70 Cay $(G, S)$ is an anti-2-hasse diagram (that is, transitive) if and only if $S^{2} \subseteq S$.

Theorem 7.71 Cay $(G, S)$ is an anti-k-quasi-hasse diagram if and only if there exists an integer $i(2 \leq i \leq n)$, such that $S^{i} \subseteq S \cup S^{-1}$.

Corollary 7.72 Cay $(G, S)$ is an anti-2-quasi-hasse diagram (that is, quasi-transitive) if and only if $S^{2} \subseteq S \cup S^{-1}$.

Theorem 7.73 The anti-k-hasse index of $\operatorname{Cay}(G, S)$ is the least positive integer $k$ such that there exists some $i$, $2 \leq i \leq k$ and $S^{i} \subseteq S$. That is,

$$
\mathscr{L}(\operatorname{Cay}(G, S))=\min \left\{k \in \mathbb{N}: S^{i} \subseteq S \text { for some } i, 2 \leq i \leq k,\right\} .
$$

Theorem 7.74 The anti-k-quasi-hasse index of $\operatorname{Cay}(G, S)$ is the least positive integer $k$ such that there exists some $i, 2 \leq i \leq k$ and $S^{i} \subseteq S \cup S^{-1}$. That is,

$$
\mathscr{M}(\operatorname{Cay}(G, S))=\min \left\{k \in \mathbb{N}: S^{i} \subseteq S \cup S^{-1} \text { for some } i, 2 \leq i \leq k,\right\} .
$$

Theorem $7.75 \mathrm{Cay}(G, S)$ is anti-k-(quasi)-hasse diagram if and only if $\operatorname{Cay}\left(G, S^{-1}\right)$ is anti-k-(quasi)-hasse diagram.

Theorem 7.76 Let Cay $(G, S)$ be a Cayley digraph with connection set $S$. Then we have the following:

$$
\begin{aligned}
\text { (i) } \mathscr{L}(\operatorname{Cay}(G, S)) & =\mathscr{L}\left(\operatorname{Cay}\left(G, S^{-1}\right)\right) \\
\text { (ii) } \mathscr{M}(\operatorname{Cay}(G, S)) & =\mathscr{M}\left(\operatorname{Cay}\left(G, S^{-1}\right)\right) .
\end{aligned}
$$

\section{Acknowledgments}

The authors would like to thank the referee for his valuable suggestions which has helped us to improve this paper.

\section{References}

Bang-Jensen, J., \& Hauang, J. (1995). Quasi-transitive digraphs. J. Graph Theory, 20, 141-161. http://dx.doi.org/10.1002/jgt.3190200205

Bang-Jensen, J., \& Hauang, J. (1998). Kings Quasi-transitive digraphs. Discrete Math., 185, 19-27. http://dx.doi.org/10.1016/S0012-365X(97)00179-9

Dobson, E. (2006). Automorphism groups of metacirculant graphs of order a product of two distinct primes. Combinatorics, Probability and Computing, 15, 105-130. http://dx.doi.org/10.1017/S0963548305007066

Fraleigh, J. B. (2008). A First course in abstract algebra. Pearson education. 
Galeana-Sanchez, H., \& Cesar, Hernandez-Cruz. (2011). $k$-kernels in generalizations of transitive digraphs. Prel. Inst. Mat, UNAM, 899, 12.

Galeana-Sanchez, H., \& Cesar, Hernandez-Cruz. (2011), $k$-kernels in $k$-transitive and $k$-quasi-transitive digraphs. Prel. Inst. Mat, UNAM, 897, 14.

Godsil, C., \& Gordon, R. (2001). Algebraic Graph Theory, Graduate Texts in Mathematics. New York: SpringerVerlag.

Parthasarathy, K. R. (1994). Basic Graph Theory. New Delhi: Tata-McGraw Hill Pub.

Sampathkumarachar, E., Germina, K. A., Jisha, Elizabath Joy, \& Beena, Koshy. (2010). $k$-transitive digraphs and topologies. International Mathematical Forum, 63(5), 3111-3119. 